\title{
Improvement of orbit determination for geostationary satellites with VLBI tracking
}

\author{
HUANG Yong ${ }^{1 *}$, HU XiaoGong ${ }^{1}$, ZHANG XiuZhong ${ }^{1}$, JIANG DongRong ${ }^{1}$, GUO Rui ${ }^{2}$, \\ WANG Hong ${ }^{3} \&$ SHI ShanBin ${ }^{3}$ \\ ${ }^{1}$ Shanghai Astronomical Observatory, Chinese Academy of Sciences, Shanghai 200030, China; \\ ${ }^{2}$ Beijing Global Information Application and Development Center, Beijing 100094, China; \\ ${ }^{3}$ Beijing Institute of Tracking and Telecommunications Technology, Beijing 100094, China
}

\begin{abstract}
China's COMPASS satellite navigation system consists of five or more geostationary (GEO) satellites. The roles of GEO satellites are to improve the regional user's positioning accuracy and provide the continuous Radio Determination Satellite Service. The motion of GEO satellites relative to a ground tracking station is almost fixed, and regular orbit maneuvers are necessary to maintain the satellites' allocated positions above the equator. These features present difficulties in precise orbit determination (POD). C-band ranging via onboard transponders and the L-band pseudo-ranging technique have been used in the COMPASS system. This paper introduces VLBI tracking, which has been successfully employed in the Chinese lunar exploration programs Chang'E-1 and Chang'E-2, to the POD of GEO satellites. In contrast to ranging, which measures distances between a GEO satellite and an observer, VLBI is an angular measurement technique that constrains the satellite's position errors perpendicular to the satellite-to-observer direction. As a demonstration, the Chinese VLBI Network organized a tracking and orbit-determination experiment for a GEO navigation satellite lasting $24 \mathrm{~h}$. This paper uses the VLBI delay and delay-rate data, in combination with C-band ranging data, to determine the GEO satellite's orbit. The accuracies of the VLBI delay and delay rate data are about $3.6 \mathrm{~ns}$ and $0.4 \mathrm{ps} / \mathrm{s}$, respectively. Data analysis shows that the VLBI data are able to calibrate systematic errors of the C-band ranging data, and the combination of the two observations improves orbit prediction accuracy with short-arc data, which is important for orbital recovery after maneuvers of GEO satellites. With the implementation of VLBI2010, it is possible for VLBI to be applied in the COMPASS satellite navigation system.
\end{abstract}

geostationary satellites, very long baseline interferometry, orbit determination

Citation: Huang Y, Hu X G, Zhang X Z, et al. Improvement of orbit determination for geostationary satellites with VLBI tracking. Chinese Sci Bull, 2011, 56: 2765-2772, doi: 10.1007/s11434-011-4647-0

The Global Positioning System (GPS) constellation consists of more than 24 Medium Earth Orbit (MEO) satellites on six orbital planes [1], and China's COMPASS satellite navigation system will deploy a constellation consisting of three Geostationary Earth Orbit (GEO) satellites, three Inclined Geostationary Earth Orbit (IGSO) satellites and 24 MEO satellites [2] within 2 years. The roles of GEO satellites are to improve the regional user's positioning accuracy, and provide the continuous Radio Determination Satellite

*Corresponding author (email: yongh@ @shao.ac.cn)
Service (RDSS). Compared with orbits of MEO and IGSO satellites, the orbits of GEO satellites are more difficult to determine because (1) the observation geometry is rather poor owing to the limitation regional distribution of the tracking network, (2) the relative motion of GEO satellites to a ground station is almost fixed, which results in biases in ranging data that cannot be effectively estimated owing to the weakness of dynamic equations for GEO satellites in a rotational Earth reference system, and (3) regular orbit maneuvers are necessary for GEO satellites to maintain their allocated positions above the equator, thus preventing the 
application of long-arc orbit-determination strategies. These features present difficulties in precise orbit determination (POD) [3-6].

Unified S-band (USB) ranging and the Doppler technique are usually used for GEO satellite tracking and control, with the accuracy of USB ranging data being several meters and the accuracy of Doppler data being several centimeters per second, leading to orbit determination accuracy of a few hundred meters [7,8]. More accurate measurement techniques are needed for satellite navigation systems such as COMPASS, most of which are high-precision ranging measurement techniques such as pseudo-ranging and carrierphase positioning, two-way ranging via onboard transponders and satellite laser ranging (SLR). In the case of GEO satellites, satellite and station clock errors in pseudo-range data may not be effectively estimated; therefore, time synchronization is needed to support POD. Furthermore, despite the high (millimeter level) precision of carrier-phase measurements, phase ambiguities along with clock errors must be carefully corrected. Systematic errors in C-band ranging data that are uncalibrated transmitting/receiving and transponder delays also directly affect the POD accuracy for a GEO satellite. SLR is able to achieve high precision and is almost free of bias, but it cannot be regularly employed since it requires clear weather, and measurement data are thus limited. Generally, SLR data are used to evaluate orbit accuracy, especially the position accuracy in the radial direction [9].

Theoretical studies and simulation tests have been carried out to improve POD accuracy for GEO satellites. Recently proposed tracking techniques include high-resolution angle observations, such as very long baseline interferometry (VLBI), connected-element interferometry (CEI) and the use of high-precision optical charge-coupled device cameras, and GPS-assisted GEO-satellite POD [3,10]. A new approach referred to as mirror surface projection has been proposed [3], in which the orbital plane of the GEO satellite is set as a symmetry plane (or a mirror surface), and original observation stations are projected as virtual observation stations.

VLBI, first developed in the 1960s, achieves high accuracy and high resolution and has multiple purposes, and it has already been applied in astrogeodynamics and geodesy [11]. VLBI is a useful supplement to usual radio ranging and velocity measurement techniques because VLBI has an extremely high angular resolution (on the order of submilliseconds of arc) and strongly constrains orbital errors in the transverse direction perpendicular to the line-of-sight, while the ranging observations are able to provide constraints in and around the line-of-sight direction; a joint orbit determination using both types of data is thus expected to yield improved results [8]. VLBI eliminates the need for uplink transmission and only receives satellite downlink signals. Weak downlink signals from very far away may be picked up with large antennas, and the VLBI technique is thus mainly applied in lunar and deep-space exploration [8, 12-14]. The Chinese lunar exploration programs Chang'E-1 and Chang'E-2 combine VLBI and USB techniques for lunar capture and mission orbit insertion. The combined POD accuracy, especially the short-arc POD accuracy, is much better than the accuracy achieved with the USB alone, demonstrating VLBI real-time deep-space navigation applications for the first time [8].

To meet the demands of scientific research and social and economical developments, the International VLBI Service for Astronomy and Geodesy has put forward a bold plan to advance the current VLBI technique and systems and develop the next generation of the VLBI-VLBI2010 system [15-17]. The new system will be automated and operate unattended and will be based on small (10-12 $\mathrm{m}$ in diameter), fast-moving, and mechanically reliable antennas that can be manufactured economically. Observations should be carried out over a broad, continuous frequency range, perhaps $1-14 \mathrm{GHz}$, which includes both the current S-band and X-band frequencies for backward compatibility. One interesting aspect for the development of the VLBI2010 system is the possibility to observe Global Navigation Satellite System (GNSS) signals [18]. VLBI data, like SLR data, can also verify orbit accuracy. UT1 nutation and the celestial reference frame are currently defined for VLBI, and there is no alternative for the foreseeable future [19]. VLBI, together with GNSS signals and SLR, must continue to provide the scale of the terrestrial reference frame.

The Chinese VLBI Network (CVN) has four radio antennas located at Shanghai (diameter of $25 \mathrm{~m}$ ), Beijing (50 $\mathrm{m})$, Kunming (40 m) and Urumqi $(25 \mathrm{~m})$, with a correlation system operating at the Shanghai Astronomical Observatory [12]. The CVN has been active in international VLBI research, and is involved in tracking Chang'E-1 and Chang'E-2 [8]. Additionally, the CVN participated in the international joint observation program of Japan's SELENE-1 lunar probe [12]. The CVN will also undertake the tracking task of China's first Mars exploration project, Yinghuo-1 (YH-1) [14]. Another antenna at Shanghai with a diameter of $65 \mathrm{~m}$ is currently under construction and expected to join the CVN before the end of year 2012 .

Du et al. [10] carried out simulations for GEO-satellite POD using VLBI data and concluded that the accuracy of the GEO orbit can be improved by including VLBI data. They also analyzed the applications of CEI data in the orbit determination of GEO satellites [20]. To verify the contribution of the VLBI technique to the GEO satellite POD, the CVN organized a 24-h tracking experiment for a COMPASS GEO navigation satellite, using the downlink S-band telemetry signal as the VLBI beacon. VLBI delay and delay rate data were collected. In this paper, these VLBI data are analyzed, and the orbit is determined using VLBI combining with $\mathrm{C}$-band ranging data. 


\section{Principle of VLBI and systematic error cali- bration}

Observational targets are remote radio sources in VLBI applications in astronomy and geodesy while they are artificial beacons located within limited distances in the VLBI observation of artificial celestial bodies [11,21].

According to the principle of VLBI, the time delay between the arrivals of the same wavefront at two antennae (the delay $\tau$ ) and the temporal rate of change of the time delay (the delay rate $\dot{\tau}$ ) are measured via radio signal interference [19]. Since the distances between the antennas and the beacon are not equal, the arrival times differ; hence the delay. Conventionally, the VLBI technique considers group delays and phase delays. The group delay is usually obtained with an accuracy of nanoseconds using a formula of phase/bandwidth. Beacon bandwidth is usually less than $10 \mathrm{MHz}$ for a spacecraft. To improve the group delay accuracy, multi-frequency signals can be adopted, because the frequency range between the signals corresponds to the bandwidth. The phase delay is derived by dividing by the frequency. The differential phase delay of the S-band signals was obtained with a very small error of several picoseconds for SELENE-1, and the accuracy of orbit determination was improved to several meters $[22,23]$.

In the case of MEO/GEO or higher-altitude satellites, the satellite-station distance is much larger than the baseline distance $B$ (Figure 1). According to the principle of the VLBI delay and simple geometry, the change in delay $(\Delta \tau)$ due to along-track error in position $(\Delta \varepsilon)$ can be written as

$$
\Delta \tau=\frac{\Delta \varepsilon}{c} \cdot \frac{B}{L}
$$

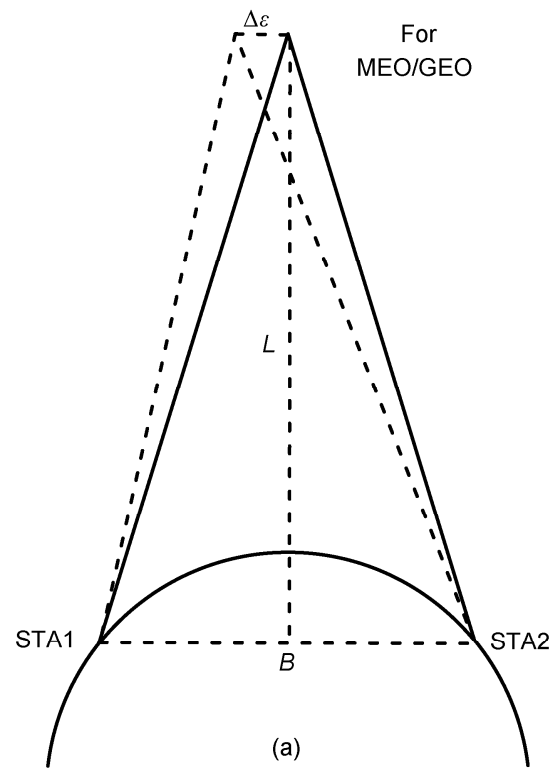

where $c$ is the speed of light, and similarly, the change in delay $(\Delta \tau)$ due to radial-track error in position $(\Delta R)$ can be written as

$$
\Delta \tau=\frac{\Delta R}{c} \cdot \frac{x^{2}}{2 L^{2}} .
$$

From eqs. (1) and (2), we see that VLBI can give planeof-sky position information more accurately than it can give line-of-sight information. For example, a delay error of $1 \mathrm{~ns}$ corresponds to an orbit error of about $4 \mathrm{~m}$ for the GEO satellite with a baseline of $3000 \mathrm{~km}$.

At least three VLBI baselines are needed for positioning. In orbit determination using a statistical method, however, even VLBI measurements for one baseline can be used. Of course, several baselines can constrain different directions of the satellite's motion, thus improving the accuracy of orbit determination.

To eliminate systematic errors relating to instruments and clocks, the differential VLBI technique is adopted. By alternately tracking an artificial VLBI beacon and a nearby extragalactic radio source (i.e. a quasar) of known location, common ground and atmospheric propagation error sources cancel out and the effects of transmission media, timing, polar motion, and station location uncertainties are reduced $[8,11,13]$. The degree of error reduction depends on the spacecraft-quasar angular separation. In practice, a quasar near the beacon is observed first, and the time delay from the quasar to different antennas, which is designated $\tau_{\mathrm{RSO}}$ and includes geometric delay $\tau_{\mathrm{RS}}$ and other error sources, is derived:

$$
\tau_{\mathrm{RSO}}=\tau_{\mathrm{RS}}+\Delta \tau_{\mathrm{RS}} .
$$

The VLBI beacon is then observed, and similarly, the

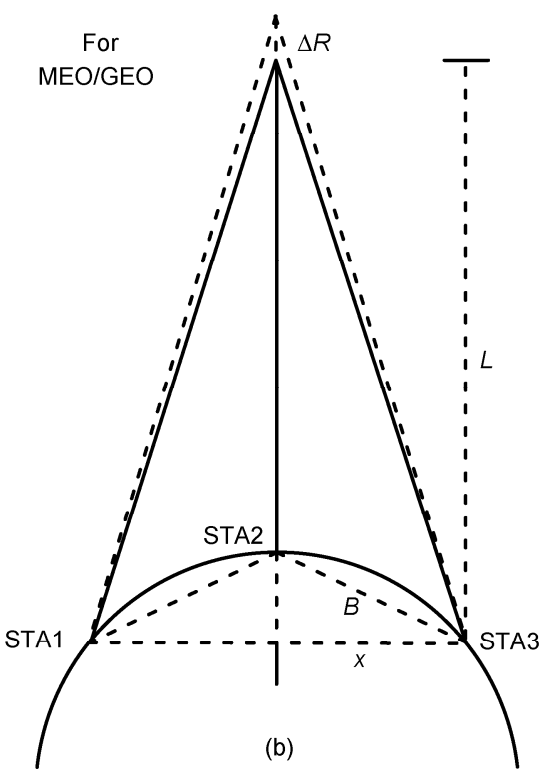

Figure 1 Diagram of how delay error relates to the position errors in radial (a) and transverse (b) directions. 
time delay of the probe is derived:

$$
\tau_{\mathrm{SCO}}=\tau_{\mathrm{SC}}+\Delta \tau_{\mathrm{SC}}
$$

where $\tau_{\mathrm{SCO}}$ and $\tau_{\mathrm{SC}}$ are the observation and geometric delays, respectively [13].

The International Celestial Reference Frame (ICRF2) uses nearly 30 years of VLBI observations at radio wavelengths, and the positional accuracy of 295 ICRF2 defining sources is better than 0.3 mas $[11,13]$. If the location of a quasar is well known, then $\tau_{\mathrm{RS}}$ is also well known, and $\Delta \tau_{\mathrm{RS}}$ can be derived from $\Delta \tau_{\mathrm{RS}}=\tau_{\mathrm{RSO}}-\tau_{\mathrm{RS}}$. Because the beaconquasar angular separation is small, we assume that $\Delta \tau_{\mathrm{SC}}$ $\approx \Delta \tau \mathrm{RS}$. The influences of the atmosphere, ionosphere and receivers are almost completely canceled out by taking the difference in the correlation phase [13].

In the real-time tracking of Chang'E-1 with VLBI observations, several quasars were observed for about $1 \mathrm{~h}$ before observations of Chang'E-1 commenced, and systematic errors such as clock errors and instrument delay errors were solved in the form of a time delay and delay rate for each of the six CVN baselines. These errors were used to correct the following observations of Chang'E-1. In each hour of observations that followed, a quasar was observed for $20 \mathrm{~min}$ and the Chang'E-1 for $40 \mathrm{~min}$. After the Chang'E-1 observation was complete, a quasar was once again observed. In post-processing, all observations of quasars were processed and the time delay and delay rate of Chang'E-1 were recalibrated.

The accuracy of the quasar calibration directly affects the real-time VLBI data. For example, calibration error of 1 $\mathrm{ps} / \mathrm{s}$ induces error in the time delay of $3.6 \mathrm{~ns}$ after about $1 \mathrm{~h}$. In the case of tracking Chang'E-2, the systematic delay rate was estimated not on the basis of quasar calibration but on the frequency rate of the atomic clocks at the four antennas as determined by GPS timing, whose differentials are equivalent to the delay rate. Normally, the behavior of the hydrogen atomic clock is very stable and the accuracy of long-term stability is about $10^{-15}-10^{-14}$. The results show that this modified strategy of estimating the systematic delay rate performs well and the accuracy of real-time VLBI data is improved, especially for time delay data. This calibration method is used in the VLBI tracking experiment for the GEO satellite in this paper.

\section{Data analysis and discussion}

The CVN organized a GEO-satellite tracking and orbit determination experiment for August 26, 2010. The GEO satellite was located at latitude of $84^{\circ}$ east. The satellite signal frequency was approximately $2.2 \mathrm{GHz}$, and the bandwidth was $2 \mathrm{MHz}$. The CVN tracked the GEO satellite for about $24 \mathrm{~h}$, and obtained tracking data of the VLBI delay and delay rate. The longest baseline of about $3249 \mathrm{~km}$ was
Shanghai-Urumqi in an east-west direction, and the shortest baseline of about $1114 \mathrm{~km}$ was Shanghai-Beijing. Each CVN station was equipped with a low temperature receiver at S/X wave band, VLBI data acquisition terminals and a hydrogen atomic clock. The correlation system at Shanghai processed VLBI observations. The VLBI data sampling rate was approximately once every $5 \mathrm{~s}$, and the noise level of the VLBI delay and delay rate were approximately $1 \mathrm{~ns}$ and 0.2 ps/s, respectively.

C-band ranging data were also available for the GEO satellite. The tracking system was established by the National Time Service Center, Chinese Academy of Sciences [24,25]. The ground tracking network consisted of four domestic stations. The accuracy of the $\mathrm{C}$-band ranging data was several centimeters. The tracking system can be used for routine observations since it is not affected by weather, and the observing system is very stable.

Pseudo-range and carrier phase measurements are usually made in the POD of a navigation satellite. For GEO satellites, however, the clock and phase ambiguity errors cannot be effectively determined, and time synchronization is needed before orbit determination. These data are not analyzed in this paper.

In this paper, VLBI data and C-band ranging data are analyzed. First, the orbit is determined jointly using two types of data, and the biases of ranging data are solved. We then analyze POD using only ranging data, focusing on the effects of different systematic errors, and POD using only VLBI data is also analyzed. Finally, short-arc orbit determination and prediction are analyzed in relation to the requirements of rapid orbit recovery after GEO satellite maneuvers.

The principle of statistical orbital determination is implemented in the data processing, with the adopted astronomical constants, reference system, and dynamical and measurement models referred to IERS96.

The dynamical perturbation models are (1) three-body gravitational attractions of the Sun and Moon, (2) gravitational attractions of solid-Earth tides, (3) gravitational attractions of oceanic tides, (4) gravitational attractions of the non-spherical Earth (Earth gravity model JGM3, truncated to the 10th degree and order), (5) radiation pressure of the Sun, (6) relativistic perturbation, (7) perturbation of the Earth's rotational deformation, and (8) empirical radialtransverse-normal accelerations.

The measurement models are (1) Saastamoinen-NMF's atmospheric refraction, (2) the displacement of the station due to the solid-Earth tide, (3) the effects of the secular tidal term on the station coordinates, (4) the effects of the loading of the oceanic tides on station coordinates, and (5) the effect of the Earth's rotational deformation on station coordinates.

The reference systems are (1) the mean equator $(x-y$ plane) and mean spring equinox (the direction of the $x$-axis) at J2000.0, (2) ITRF2000 for the coordinates of the stations and their velocities of motion, (3) the precession model 
IAU1976, (4) the 1980 nutation model and IERS nutation correction, and (5) the planetary ephemeris DE403/LE403.

A 24-h arc orbit is considered, and estimated parameters are six orbital elements, one solar radiation pressure parameter, constant ranging biases at individual ranging stations, and constant accelerations in the transverse direction (once every $8 \mathrm{~h}$ ).

For C-band ranging, there are systematic errors in station transmission and receiving systems and the satellite transponder delay. The satellite transponder delay is the same at each station and stable over the course of $1 \mathrm{~d}$ [6]. Given the strong parameter correlation, systematic errors of the station equipment and satellite transponder cannot be separated for GEO satellites; therefore, the ranging bias includes all these errors in this case. Because of the weakness of the GEO-satellite dynamic equations in a rotational Earth reference system, the ranging bias and orbital elements are strongly correlated. To prevent the weakening of orbit estimation with incorporation of bias estimates for each station, a corrective measure was taken assuming that the sum of all ranging biases is zero [25]. A new strategy of GEO-satellite POD is proposed by combining two tracking techniques: SLR and C-band transfer ranging. An accurate and effective approach for ranging bias calibration was implemented by Guo et al. [7]. In this work, the VLBI data are also used to calibrate the bias in C-band ranging data.

Figures 2 and 3 are the time series of the POD residuals of ranging and VLBI data. The root-mean-square (RMS) of $\mathrm{C}$-band ranging data is about $0.19 \mathrm{~m}$. The RMS of the VLBI delay and that of the delay rate data are $3.6 \mathrm{~ns}$ and $0.42 \mathrm{ps} / \mathrm{s}$, respectively.

Figure 2 shows different noise levels for ranging data at the four stations, and the accuracies for stations 1 and 4 are lower than those for the other two stations. The VLBI antennas observed a radio source during the blank parts of the plots in Figure 3 to correct for systematic errors. The time series of residuals are flat and small for all observation arcs except for baselines relating to Kunming, which may be due

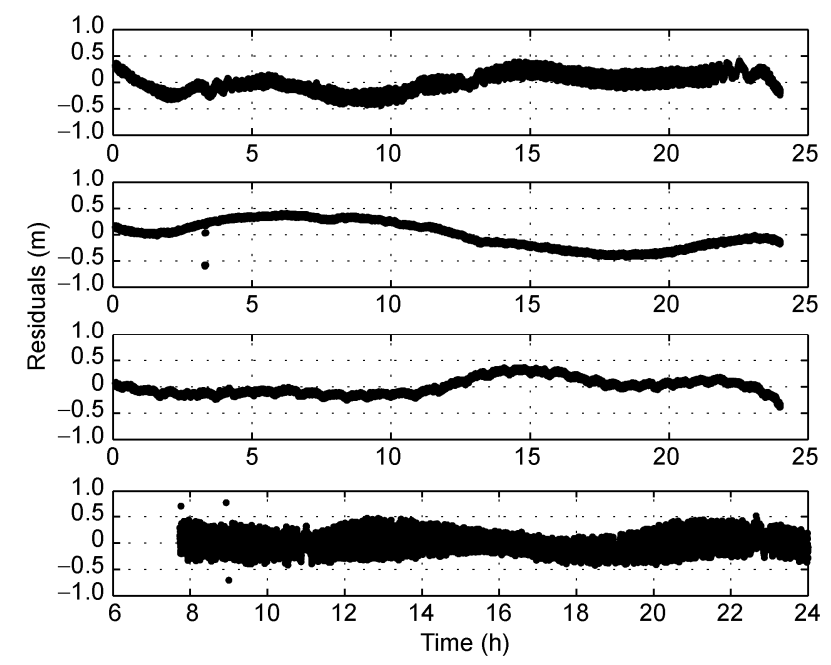

Figure 2 Residuals of ranging data after combined POD. to the low accuracy of quasar calibration. There are no VLBI data related to Kunming between 12-14 h owing to instrumental problems at Kunming. In general, VLBI observations achieved the desired accuracy in spite of some observational problems.

The orbit is then solved using only C-band ranging data with two strategies. Ranging bias is solved in strategy 1 , and the orbital difference with the combined POD is shown in Figure 4(a). The difference is about $60 \mathrm{~m}$ in the transverse direction, and the corresponding VLBI delay error is about 10-20 ns, resulting from the strong correlation between ranging bias and orbit elements. Other analyses show that different a priori values of ranging bias also affect the solved orbit, mainly in the transverse direction. Instead of solving for range bias, strategy 2 fixes the ranging bias obtained from combined POD. The orbital difference with the combined POD is shown in Figure 4(b), and it is seen that the difference is less than $10 \mathrm{~m}$ in three dimensions. Figure 4 also shows that the orbital accuracy achieved with only $\mathrm{C}$-band ranging data is higher in the radial direction, and the error is mainly in the other two directions.

We also determine the orbit using only VLBI data. The positional difference with combined POD is about $15 \mathrm{~m}$. Figure 5 shows the orbital difference, and we see that the radial difference is large, about $10 \mathrm{~m}$, probably because of the weak constraint of VLBI data in the radial direction.

It has been found that the accuracy of the GEO orbit can be improved by including a small quantity of VLBI data [10]. Therefore, we also use 3-h VLBI data combined with 24-h ranging data for the calculation. The result shows that the accuracy does not decrease significantly with only little VLBI data, confirming the reference [10].

The above analyses show that VLBI data can be used to calibrate ranging bias, and VLBI data are able to strongly constrain the orbit in the transverse directions, while the ranging observations provide a stronger constraint in and around the line-of-sight direction. Joint orbit determination using both data types is expected to yield improved orbital accuracy. Similarly, when the orbit of the GEO satellite is determined using pseudo-range measurements, VLBI data can also be used to calibrate clock errors in pseudo-range data after clock errors are effectively modeled [26].

Although GEO satellites are deployed as COMPASS navigation satellites, it is difficult to precisely model the forces of frequent GEO-satellite maneuvers. The predicted orbit may soon be invalid after a maneuver commences owing to maneuver forces, and the actual orbit is sometimes more than several kilometers from the predicted orbit if the maneuver is not considered. Prediction of the orbit with short-arc observations by reducing the number of estimated parameters after the maneuver operation is complete has been proposed [6]. It is assumed that the common system bias and biases of individual stations are constant and can be obtained from orbit determination with long-arc observations before the maneuver. In this way, only six orbit 

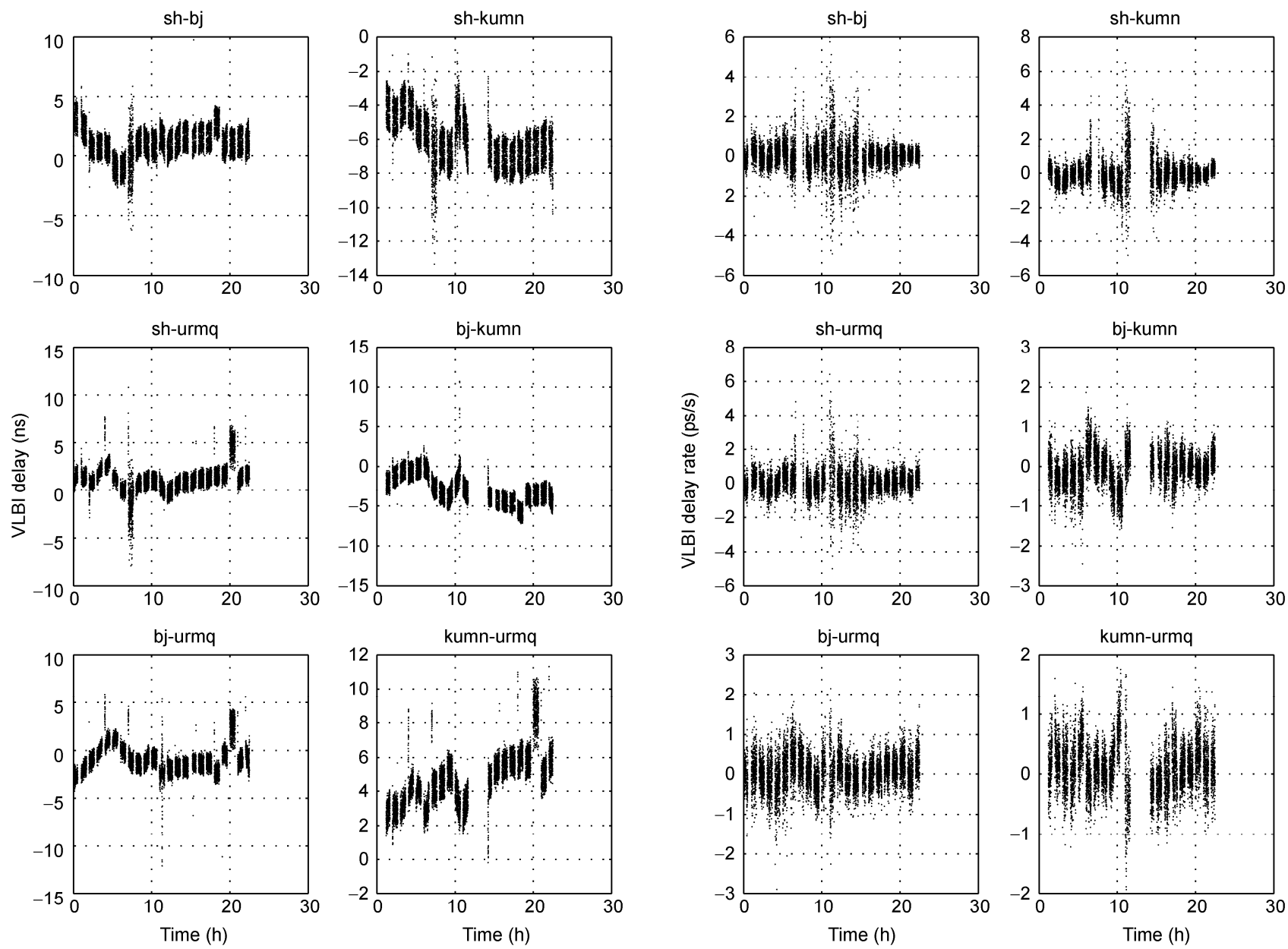

(a)
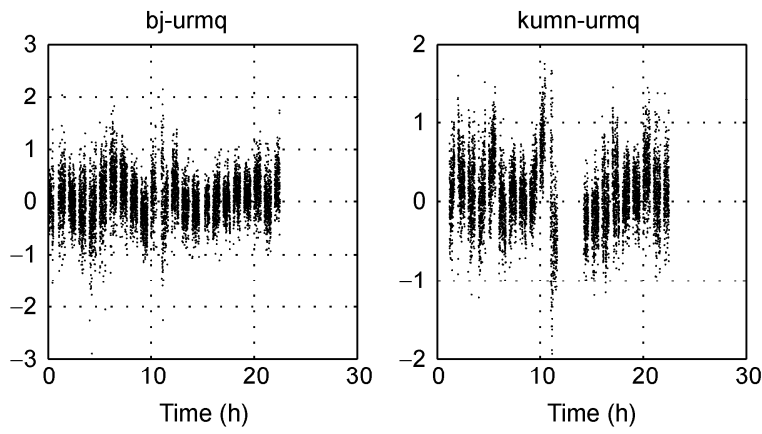

(b)

Figure 3 Residuals of VLBI data after combined POD. (a) The VLBI delay; (b) the VLBI delay rate.

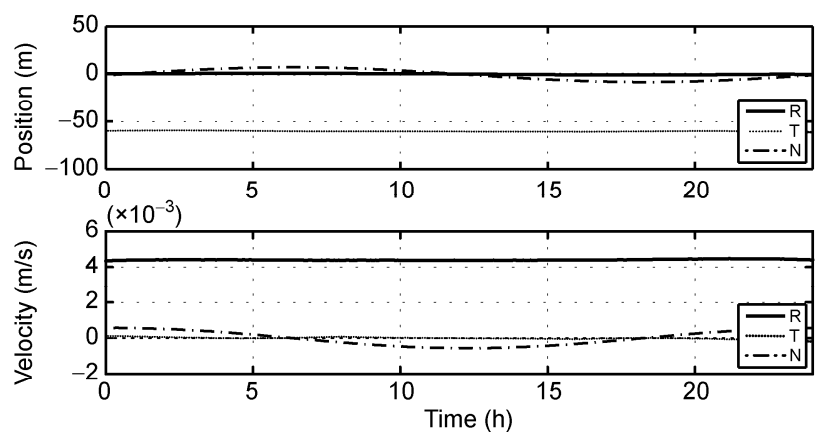

(a)
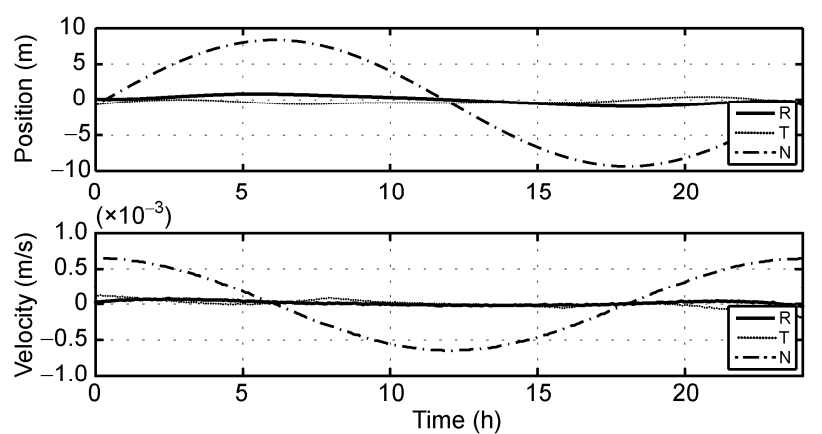

(b)

Figure 4 Orbital differences between POD using only C-band ranging data and combined POD in the radial, transverse and normal directions. (a) Solving for ranging bias; (b) fixing ranging bias. The upper and lower graphs present position and velocity differences, respectively.

elements are solved, and an accurate ephemeris is soon obtained.

This paper develops a different approach in which the orbit is determined and predicted with a combination of short-arc VLBI and C-band ranging data. The observation arc is only $15 \mathrm{~min}$ and the orbit is predicted for $12 \mathrm{~h} \mathrm{ac}$ cording to the actual situation. The residual of ranging data relative to the predicted orbit is calculated as the accuracy evaluation of the predicted orbit in the radial direction. Using VLBI and C-band ranging data, the predicted orbit accuracy is about $1.2 \mathrm{~m}$ (RMS of residuals) over a period of $2 \mathrm{~h}, 4.4 \mathrm{~m}$ over a period of $6 \mathrm{~h}$, and $7.0 \mathrm{~m}$ over a period of $12 \mathrm{~h}$. Meanwhile, using only C-band ranging data, the predicted accuracy is about $1.0,8.1$ and $18.8 \mathrm{~m}$, respectively. Results are presented in Table 1 and Figure 6 . These results show that VLBI data can be used to improve short-arc POD 

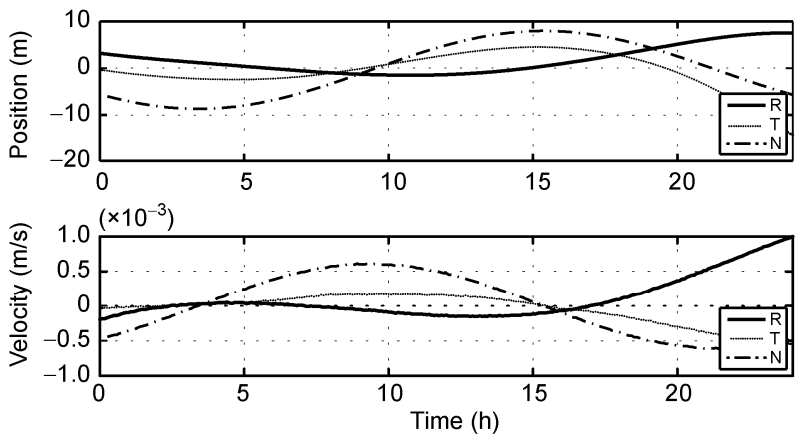

Figure 5 Orbital differences between POD using only VLBI data and combined POD.

Table 1 RMS of O-C of the predicted orbit with a 15-min short-arc observation (unit: $\mathrm{m}$ )

\begin{tabular}{lllr}
\hline & $2 \mathrm{~h}$ & $6 \mathrm{~h}$ & $12 \mathrm{~h}$ \\
\hline Only ranging data & 1.0 & 8.1 & 18.8 \\
VLBI+ranging data & 1.2 & 4.4 & 7.0 \\
\hline
\end{tabular}
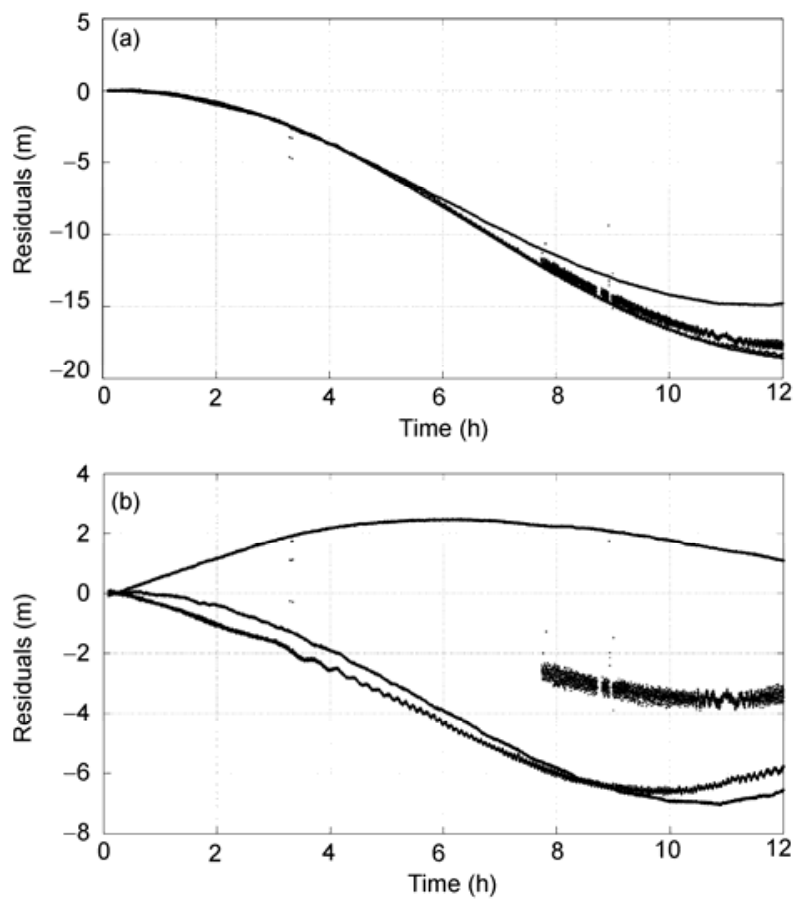

Figure 6 Accuracy of orbit determination (first $15 \mathrm{~min}$ in the plots) and prediction as evaluated by O-C. (a) POD using C-band ranging data only; (b) combined POD.

accuracy for the rapid orbit recovery of GEO satellites.

\section{Conclusions}

For many years, Earth-orbiting and interplanetary spacecrafts have been navigated using conventional radio metric measurements. VLBI is a useful complement to the usual radio ranging and velocity measurements. VLBI can calibrate systematic errors of other techniques, and verifies the orbit accuracy just like SLR. VLBI has extremely high angular resolution and provides the strongest constraint on the orbit in transverse directions. Joint orbit determination is expected to yield improved results.

In this paper, a GEO satellite tracking experiment organized by the CVN is presented and VLBI data are analyzed. The orbit is determined combining VLBI with C-band ranging data. The accuracy of VLBI delay data is limited to about 3 ns owing to the narrow bandwidth and limitations of beacon design, and the corresponding orbit error is about $10 \mathrm{~m}$ for the GEO satellite. The contribution of VLBI to long-arc POD is limited, and the accuracy of the combined POD is about $10 \mathrm{~m}$. Meanwhile VLBI data are able to precisely calibrate range bias and improve the predicted-orbit accuracy for the rapid orbit recovery of GEO satellites. The accuracy of VLBI data may be further improved to $0.1 \mathrm{~ns}$ or better by adopting multi-frequency beacon designs or increasing the bandwidth. For example, with a specially designed VLBI beacon, a noise level better than $0.1 \mathrm{~ns}$ for VLBI delay data has been achieved for the Chang'E-2 program.

VLBI has been employed in deep-space exploration for the first and second Chinese lunar exploration programs and for the Japanese SELENE-1 program, and will also be used in future deep-space exploration projects such as Mars missions. With the development of the VLBI technique, especially with the development of a network of VLBI2010 standards, VLBI may also be applied to satellite navigation systems.

The authors would like to thank the CVN for providing tracking data, and QIAN ZhiHan, LIU QingHui, ZHENG WeiMin, LI JinLing, PING JinSong, WANG GuangLi, SHU FengChun and HONG XiaoYu for their comments and suggestions. This work was supported by the National Natural Science Foundation of China (10703011, 11073047, 11033004), the National High-Tech Research and Development Program of China (2008AA12A209, 2008AA12A210), and the Science and Technology Commission of Shanghai (06DZ22101).

1 Liu J Y, Li Z H, Wang Y H, et al. Principle and Application of Global Positioning System (in Chinese). Bejing: Surveying and Mapping Press, 1993

2 Zhou S S, Hu X G, Wu B. Orbit determination and prediction accuracy analysis for a regional tracking network. Sci China Phys Mech Astron, 2010, 53: 1130-1138

3 Ou J K, Liu J H, Sun B Q, et al. Precision orbit determination of a geostationary satellite GEO with mirror surface projection method (in Chinese). Geomat Inform Sci Wuhan Univ, 2007, 32: 975-979

4 Du L. A study of the precise orbit determination of geostationary satellites (in Chinese). Dissertation for the Doctoral Degree. Zhengzhou: Information Engineering University, 2006

5 Huang Y, Hu X G, Huang C, et al. Precise orbit determination of a maneuvered GEO satellite using CAPS ranging data. Sci China Ser G Phys Mech Astron, 2009, 52: 346-352

6 Yang X H, Li Z G, Feng C G, et al. Methods of rapid orbit forecasting after maneuvers for geostationary satellites. Sci China Ser G Phys Mech Astron, 2009, 52: 333-338 
7 Guo R, Hu X G, Tang B, et al. Precise orbit determination for geostationary satellites with multiple tracking techniques. Chinese Sci Bull, 2010, 55: 687-692

8 Yan J G, Ping J S, Li F, et al. Chang'E-1 precision orbit determination and lunar gravity field solution. Adv Space Res, 2010, 46: 50-57

9 Guo R, Hu X G, Liu L, et al. Orbit determination for geostationary satellites with the combination of transfer ranging and pseudorange data. Sci China Phys Mech Astron, 2010, 53: 1746-1754

10 Du L, Zheng Y, Li J. VLBI2 augmented orbit determination for geostationary satellites (in Chinese). J Zhengzhou Inst Survey Map, 2006, 23: 269-271

11 Ye S H, Huang C. Astro-Geodynamics (in Chinese). Jinan: Shandong Science \& Technology Press, 1981

12 Hong X Y. VLBI techniques and application in the Chang'E lunar orbiter (in Chinese). Nat Mag, 2007, 29: 297-299

13 Zhu X Y, Li C L, Zhang H B. A survey of VLBI technique for deep space exploration and trend in China current situation and development (in Chinese). J Astron, 2010, 31: 1893-1899

14 Cao J F, Huang Y, Hu X G, et al. Mars express tracking and orbit determination trials with Chinese VLBI network. Chinese Sci Bull, 2010, 55: 3654-3660

15 Pet R B, Niell A, Behrend D, et al. Design aspects of the VLBI2010 system. IVS Annual Report, USA, 2009

16 Li J L, Wei E H, Sun Z M, et al. Discussion on future configuration design of Chinese astrometric and geodetic VLBI network (in Chinese). Geomat Inform Sci Wuhan Univ, 2010, 35: 670-673

17 Xia J C, Li J L, Han Y B. On the next generation of the VLBI system-VLBI2010 (in Chinese). Prog Geophys, 2010, 25: 1623-1627
18 Tornatore V, Haas R. Considerations on the observation of GNSSsignals with the VLBI2010 system. In: Proceedings of the 19th European VLBI for Geodesy and Astrometry Working Meeting, Bordeaux, 2009

19 Hu X G, Huang C, Qian Z H. Space VLBI and its applications in astrogeodynamics (in Chinese). Prog Astron, 1998, 16: 177-186

20 Du L, Zheng Y, Zhang H W, et al. Phase ambiguity and location of tracking sites of orbit determination of GEO satellite (in Chinese). J Zhengzhou Inst Survey Map, 2003, 20: 100-102

21 Ping J S. The research on observation of spacecrafts by VLBI in the solar system (in Chinese). Dissertation for the Doctoral Degree. Shanghai: Shanghai Astronomical Observatory, Chinese Academy of Sciences, 1996

22 Liu Q H, Shi X, Kikuchi F, et al. High-accuracy same-beam VLBI observations using Shanghai and Urumqi telescopes. Sci China Ser G Phys Mech Astron, 2009, 52: 1858-1866

23 Goossens S, Matsumoto K, Ishihara Y, et al. Results for orbit determination of the three satellites of Kaguya. J Geod Soc Jpn, 2009, 55: 255-268

24 Ai G X, Shi H L, Wu H T, et al. The principle of positioning system based on communication satellites. Sci China Ser G Phys Mech Astron, 2009, 52: 472-488

$25 \mathrm{Li}$ Z G, Yang X H, Ai G X, et al. A new method for determination of satellite orbits by transfer. Sci China Ser G Phys Mech Astron, 2009, 52: 384-392

26 Hu X Y, Huang Y, Hu X G. On the second order clock bias model in orbit determination for the MEO satellite (in Chinese). J Astron, 2009, 30: 924-929

Open Access This article is distributed under the terms of the Creative Commons Attribution License which permits any use, distribution, and reproduction in any medium, provided the original author(s) and source are credited. 